Cite this: Phys. Chem. Chem. Phys., 2014, 16, 4830

Received 3rd December 2013, Accepted 14th January 2014

DOI: $10.1039 / c 3 c p 55103 b$

www.rsc.org/pccp

\title{
Thermal degradation kinetics and decomposition mechanism of PBSu nanocomposites with silica-nanotubes and strontium hydroxyapatite nanorods
}

\author{
D. G. Papageorgiou, ${ }^{a}$ E. Roumeli, ${ }^{a}$ K. Chrissafis, ${ }^{a}$ Ch. Lioutas, ${ }^{a}$ K. Triantafyllidis, ${ }^{b}$ \\ D. Bikiaris ${ }^{\star b}$ and A. R. Boccaccini ${ }^{c}$
}

\begin{abstract}
Novel poly(butylene succinate) (PBSu) nanocomposites containing 5 and 20 wt\% mesoporous strontium hydroxyapatite nanorods (SrHNRs) and silica nanotubes (SiNTs) were prepared by melt-mixing. A systematic investigation of the thermal stability and decomposition kinetics of PBSu was performed using pyrolysis-gas chromatography-mass spectroscopy (Py-GC-MS) and thermogravimetry (TG). Thorough studies of evolving decomposition compounds along with the isoconversional and model-fitting analysis of mass loss data led to the proposal of a decomposition mechanism for PBSu. Moreover, the effects of SrHNRs and SiNTs on the thermal stability and decomposition kinetics of PBSu were also examined in detail. The complementary use of these techniques revealed that the incorporation of SiNTs in PBSu does not induce significant effects neither on its thermal stability nor on its decomposition mechanism. In contrast, the addition of SrHNRs resulted in the catalysis of the initial decomposition steps of PBSu and also in modified decomposition mechanisms and activation energies. The evolving gaseous products of PBSu and their evolution pattern in the SiNT nanocomposites were the same as in neat PBSu, while they were slightly modified for the SrHNR nanocomposites, confirming the findings from thermogravimetric analysis.
\end{abstract}

\section{Introduction}

During the last few decades, a variety of polymeric materials have been tested for various biomedical applications and especially bone tissue engineering. ${ }^{1-3}$ The fundamental goal of tissue engineering is the development of biocompatible substitutes that will restore, maintain or improve tissue functions. $\mathrm{PBSu}$ is an aliphatic biodegradable thermoplastic polyester, which is synthesized through condensation polymerization of 1,4-butanediol and succinic acid. ${ }^{4,5}$ PBSu presents interesting properties such as biodegradability, processability and chemical resistance which are the reasons for its use in numerous applications. However, PBSu is not free from disadvantages, since it presents low stiffness and poor mechanical and thermal properties. ${ }^{6,7}$

The introduction of filler particles into a polymeric matrix is an effective way of improving various physicochemical properties

\footnotetext{
${ }^{a}$ Solid State Physics Department, School of Physics, Aristotle University of Thessaloniki, 54124 Thessaloniki, Macedonia, Greece

${ }^{b}$ Department of Chemistry, Aristotle University of Thessaloniki, GR-541 24, Thessaloniki, Macedonia, Greece. E-mail: dbic@chem.auth.gr; Fax: +30 2310 997667; Tel: +30 2310997812

${ }^{c}$ Institute of Biomaterials, Department of Materials Science and Engineering,

University of Erlangen-Nuremberg, Cauerstrasse 6, 91058 Erlangen, Germany
}

of the pristine polymer. ${ }^{8}$ Consequently, polymer nanocomposites have found application in drug delivery systems and bone tissue engineering. ${ }^{9-12}$ In the current study strontium hydroxyapatite nanorods (SrHNRs) and silica nanotubes (SiNTs) were prepared in order to be inserted into a PBSu matrix. The SrHNRs with mesoporous structure were used previously as a drug carrier for controlled release. ${ }^{13}$ Furthermore, a lot of drugs containing strontium salts such as strontium ranelate help new bone tissue to grow and decrease bone loss. Additionally, silica is known to recalcify and strengthen bone tissue by increasing the mineral density and reducing resorption along with promoting osteoblast-like behavior. ${ }^{14,15}$ Thus, the unique geometry and the significant properties of silica nanotubes can enhance the properties of PBSu. The particular nanofillers are not commercially available and have been synthesized in the present work from our group, while such nanocomposites have been prepared and studied for the first time in the literature.

However, it is well known that nanoparticles, especially those having surface reactive groups like our nanofillers, can affect the decomposition rate of polymers and also in some cases to alter the decomposition mechanism. ${ }^{16,17}$ Furthermore, the rate and degradation mechanism of nanocomposites are 
known to affect many important cellular processes such as cell growth, tissue regeneration and cell response. ${ }^{15}$ In the case of biocompatible polyesters like PBSu some of the produced byproducts could also be toxic. For this reason, in the current work a detailed study of the mechanism of degradation of PBSu-SrHNRs and PBSu-SiNTs nanocomposites has been conducted. The gas chromatography-mass spectroscopy technique has been applied simultaneously with the study of thermal decomposition kinetics for the evaluation of the effect of SiNTs and SrHNRs on PBSu decomposition. Furthermore, the collected decomposition compounds and their identification with mass spectroscopy of each sample were reported for the first time in the literature.

\section{Experimental}

\subsection{Materials}

For the preparation of PBSu succinic acid (SA, purum 99\%), 1,4butanediol (purum 99\%) and tetrabutoxytitanium $\left[\mathrm{Ti}(\mathrm{OBu})_{4}\right]$ as a catalyst (analytical grade) were used. For preparation of strontium hydroxyapatite nanorods $\left[\mathrm{Sr}_{5}\left(\mathrm{PO}_{4}\right)_{3} \mathrm{OH}\right]$, strontium nitrate $\left[\mathrm{Sr}\left(\mathrm{NO}_{3}\right)_{2}\right]$, trisodium citrate (labeled as $\left.\mathrm{Cit}^{3-}\right),\left(\mathrm{NH}_{4}\right)_{2} \mathrm{HPO}_{4}$, and cetyl-trimethyl-ammonium bromide (CTAB) as a surfactant (analytical grade) were used. For preparation of $\mathrm{SiO}_{2}$ nanotubes tetraethoxy silicon oxide (TEOS) and the $n$-dodecylamine hydrochloride surfactant as a template were used. All these materials were purchased from Aldrich Chemical Co.

\subsection{Preparation of poly(butylene succinate)}

PBSu was prepared by the two-stage melt polycondensation method (esterification and polycondensation) in a glass batch reactor. The proper amount of succinic acid and 1,4-butanediol in an acid/diol molar ratio $1 / 1.1$ and the catalyst $\left[\mathrm{Ti}(\mathrm{OBu})_{4}\right](1 \times$ $10^{-3} \mathrm{~mol} / \mathrm{mol} \mathrm{SA}$ ) were charged into the reaction tube of the polyesterification apparatus. The apparatus with the reagents was evacuated several times and filled with argon in order to completely remove oxygen. The reaction mixture was heated at $190{ }^{\circ} \mathrm{C}$ under an argon atmosphere and constant speed stirring (350 rpm). This first step (esterification) is considered to be completed after the collection of a theoretical amount of $\mathrm{H}_{2} \mathrm{O}$ (about $3 \mathrm{~h}$ ), which was removed from the reaction mixture by distillation and collected in a graduated cylinder. In the second step of polycondensation vacuum (5.0 Pa) was applied slowly over a period of $15 \mathrm{~min}$, to avoid excessive foaming and minimize oligomer sublimation, a potential problem during melt polycondensation. The temperature was slowly increased to $220{ }^{\circ} \mathrm{C}$ while stirring speed was increased to $720 \mathrm{rpm}$. The polycondensation continued for about $60 \mathrm{~min}$ at $220{ }^{\circ} \mathrm{C}$ and after that time the temperature was increased to $240{ }^{\circ} \mathrm{C}$ and the reaction was continued for $60 \mathrm{~min}$.

\subsection{Preparation of mesoporous strontium hydroxyapatite nanorods}

In a typical procedure for the preparation of luminescent $\mathrm{Sr}_{5}\left(\mathrm{PO}_{4}\right)_{3} \mathrm{OH}$ nanorods $(\mathrm{Sr}), 3 \mathrm{mmol}$ of $\mathrm{Sr}\left(\mathrm{NO}_{3}\right)_{2}, 0.5 \mathrm{~g}$ of CTAB, and $10 \mathrm{~mL}$ of ammonia solution $\left(\mathrm{NH}_{3} \cdot \mathrm{H}_{2} \mathrm{O}\right.$ ) (used for adjusting the $\mathrm{pH}$ value to make the solution alkaline) were dissolved in $30 \mathrm{~mL}$ of deionized water to form solution 1 . Then, $6 \mathrm{mmol}$ of trisodium citrate (labeled as $\mathrm{Cit}^{3-}$, the molar ratio of $\mathrm{Cit}^{3-} / \mathrm{Sr}^{2-}$ is $2: 1$ ) and $2 \mathrm{mmol}$ of $\left(\mathrm{NH}_{4}\right)_{2} \mathrm{HPO}_{4}$ were added into $20 \mathrm{~mL} \mathrm{H}_{2} \mathrm{O}$ to form solution 2 . After vigorously stirring for $30 \mathrm{~min}$, solution 2 was introduced into solution 1 (dropwise). After additional agitation for $20 \mathrm{~min}$, the as-obtained mixed solution was transferred into a Teflon bottle $(80 \mathrm{~mL})$ held in a stainless steel autoclave, sealed, and maintained at $180{ }^{\circ} \mathrm{C}$ for $24 \mathrm{~h}$. As the autoclave cooled to room temperature naturally, the precipitate was separated by centrifugation, washed with deionized water and ethanol in sequence. Then, the obtained product was redispersed in $150 \mathrm{~mL}$ of acetone and refluxed at $80{ }^{\circ} \mathrm{C}$ for $48 \mathrm{~h}$ to remove the residual template CTAB. Finally, the precipitate was separated by centrifugation again and dried under vacuum at $70{ }^{\circ} \mathrm{C}$ for $24 \mathrm{~h}$ to obtain the final sample. ${ }^{13}$

\subsection{Preparation of $\mathrm{SiO}_{2}$ nanotubes}

Synthesis of $\mathrm{SiO}_{2}$ nanotubes was achieved by a sol-gel technique using tetraethoxy silicon oxide (TEOS) and a surfactant (n-dodecylamine hydrochloride) as a template. The necessary quantity of TEOS was dissolved in heptane $\left(\mathrm{C}_{7} \mathrm{H}_{16}\right)$ and added carefully and slowly in an aqueous solution of DAHC ( $n$-dodecylamine hydrochloride, $0.1 \mathrm{M}, \mathrm{pH}=4.5$ ) to not disturb the membrane between organic and aqueous phases. The molar ratio of $[\mathrm{TEOS}] /[\mathrm{LAHC}]$ was 4 , same as the ratio of $\left[\mathrm{H}_{2} \mathrm{O}\right] /\left[\mathrm{C}_{7} \mathrm{H}_{16}\right]$. The system was left for 7 days and afterwards the aqueous phase was collected and the product filtered and washed with deionized water. After it was dried at $80{ }^{\circ} \mathrm{C}$ for $6 \mathrm{~h}$ it was calcined at $450{ }^{\circ} \mathrm{C}$ for $6 \mathrm{~h}$ to obtain the inorganic part. $^{18,19}$

\subsection{Preparation of PBSu nanocomposites}

Nanocomposites containing 5 and $20 \mathrm{wt} \%$ of nanofillers were prepared by melt mixing in a Haake-Buchler Reomixer (model 600) with roller blades. Two different types of nanofillers were used: SiNTs and SrHNRs. Prior to melt-mixing the nanofillers were dried by heating in a vacuum oven at $130{ }^{\circ} \mathrm{C}$ for $24 \mathrm{~h}$. The two components were physically premixed before being fed in the reomixer, in order to achieve a better dispersion. Melt blending was performed at $130{ }^{\circ} \mathrm{C}$ with $30 \mathrm{rpm}$ for $5 \mathrm{~min}$. During the mixing period the melt temperature and torque were continuously recorded. Each nanocomposite after preparation was milled and placed in a desiccator to prevent any moisture absorption.

\subsection{Transmission electron microscopy (TEM) and scanning electron microscopy (SEM)}

TEM observations were carried out on selected samples using a JEOL 120CX electron microscope operating at $100 \mathrm{kV}$ and a JEOL 2011 TEM operating at $200 \mathrm{kV}$ and having a point resolution of $0.194 \mathrm{~nm}$. Specimens suitable for TEM observation were obtained by gluing crashed material on copper grids.

SEM studies were carried out using a JEOL JMS-840A scanning microscope equipped with an energy dispersive X-ray (EDX) Oxford ISIS 300 micro-analytical system. 


\subsection{Thermogravimetric analysis}

Thermogravimetric analysis was carried out using a SETARAM SETSYS TG-DTA 16/18 instrument. Samples $(5.0 \pm 0.3 \mathrm{mg})$ were placed in alumina crucibles. An empty alumina crucible was used as reference. PBSu nanocomposites were heated from ambient temperature to $550{ }^{\circ} \mathrm{C}$ in a $50 \mathrm{~mL} \mathrm{~min}{ }^{-1}$ flow of $\mathrm{N}_{2}$ at heating rates of 5,10 and $20{ }^{\circ} \mathrm{C} \mathrm{min}{ }^{-1}$. Continuous recordings of sample temperature, sample weight and heat flow were performed.

\subsection{Pyrolysis-gas chromatography-mass spectroscopy (Py-GC-MS)}

For Py-GC-MS analysis of PBSu and its nanocomposites a very small amount of each material is placed initially into the Multi-Shot EGA/PY-3030D Pyrolyzer (Frontier Laboratories Ltd, Fukushima, Japan). For pyrolysis analysis (flash pyrolysis) the sample is placed into the sample cup which afterwards falls free into the Pyrolyzer furnace. The pyrolysis temperature is pre-selected to 3 different temperatures like 330, 390 and $500{ }^{\circ} \mathrm{C}$ and the GC oven temperature is programmed from 50 to $300{ }^{\circ} \mathrm{C}$ at $20{ }^{\circ} \mathrm{C} \mathrm{min}^{-1}$. The sample vapours generated in the furnace are split (at a ratio of $1 / 50$ ), a portion going into the column at a flow rate of $1 \mathrm{~mL} \mathrm{~min}^{-1}$ and a portion exiting the system via the vent. The pyrolyzates are separated in the Ultra Alloy metal capillary column (UA + 5) and analyzed using the MS detector GC-MS-QP2010 Ultra (Shimadzu).

\section{Kinetic methods for thermogravimetric analysis}

The rate equation which describes the degradation kinetics of a material includes the extent of conversion $(\alpha)$, the rate constant $k(T)$, the temperature $T$ and the reaction model related to the degradation mechanism $f(\alpha)$ and is given by:

$$
\frac{\mathrm{d} \alpha}{\mathrm{d} t}=k(T) f(\alpha)
$$

The temperature dependence of the rate constant is expressed in terms of the Arrhenius equation as:

$$
k=A \exp \left(-\frac{E_{\alpha}}{R T}\right)
$$

where $A$ is a frequency factor corresponding to the incidence of molecular collisions that should be obtained to produce a chemical reaction and is named pre-exponential factor, $R$ is the gas constant and $E$ is the activation energy $\left(\mathrm{kJ} \mathrm{mol}^{-1}\right)$. Generally, the reaction models for the description of complex processes such as the degradation of polymers and nanocomposites are particularly complicated, however the simplest form of $f(\alpha)$ assumes that the rate of conversion is proportional to the nth order of the material concentration:

$$
f(\alpha)=(1-\alpha)^{n}
$$

According to the isoconversional method of Friedman, ${ }^{20}$ the insertion of eqn (2) and (3) into eqn (1) and then rearrangement and taking logarithms of the parameters end up in the following equation:

$$
\ln \left(\beta \frac{\mathrm{d} \alpha}{\mathrm{d} T}\right)=\ln [A f(\alpha)]-\frac{E_{\alpha}}{R T_{\alpha}}
$$

For each $\alpha$ value, the plot of $\ln (\mathrm{d} \alpha / \mathrm{d} t)$ versus $1 / T$, obtained from thermogravimetric experiments at different heating rates, should be a straight line, whose slope gives the activation energy $E_{\alpha}$ for different degrees of conversion $\alpha$.

Another isoconversional method, which was used in the present manuscript for the calculation of the activation energy, was the integral one proposed by Kissinger, Akahira and Sunose. $^{21,22}$ It is known that the isoconversional methods demand the determination of the temperature $T$ at which a fixed fraction of the total amount of the mass is transformed. The relationship between the temperature $T$ and the heating rate $\beta$ is given by:

$$
\ln \left(\frac{\beta}{T_{\alpha}^{2}}\right)=\ln \left(\frac{A_{\alpha} R}{E_{\alpha} g(\alpha)}\right)-\frac{E_{\alpha}}{R T_{\alpha}}
$$

In order to obtain the activation energy $E$ values using the KAS method, plots of $\ln \left(\beta / T_{\alpha}^{2}\right)$ versus $1000 / T_{\alpha}$ for different values of the degree of conversion $\alpha$ are constructed and the determination of the slopes of the curves enables the calculation of $E_{\alpha}$.

\section{Results and discussion}

\subsection{Morphology of SrHNRs and SiNTs}

TEM observations were carried out for the detailed study of the morphology of SrHNRs since SEM due to the small diameter of the prepared nanorods was not appropriate to detect their morphology. Fig. 1 illustrates a typical conventional TEM bright field (BF) image of the sample showing a bundle of nanorods. The length and the diameter of the nanorods are estimated to be from $160 \mathrm{~nm}$ up to $730 \mathrm{~nm}$ with an average value of around $400 \mathrm{~nm}$ and from 12 up to $30 \mathrm{~nm}$ with a mean value of around $20 \mathrm{~nm}$ respectively. The selected area electron diffraction (SAED) pattern (upper-left inset in Fig. 1) shows characteristic concentric rings of discrete spots and reveals the polycrystalline character of the sample. The calculated d-spacings are in very good agreement with the hexagonal phase of strontium hydroxyapatite (SrHAp) (PDF No. 33-1348, space group: P63/m, No. 176). The light grey arcs pointed out in the ED pattern represent the positions of the calculated rings for this phase. Moreover the white arrow indicates the position of the most intense diffracted beams. The upper-right inset in Fig. 1 presents a magnified part of the nanorods denoted by the black orthogonal. The presence of numerous individual small white dots obviously suggests the existence of mesopores (4-5.5 nm), spreading around the surfaces of SrHNRs nanorods, which is in agreement with the literature. ${ }^{13}$

SEM micrographs of silica nanotubes showed that these are well formed and have a length of $5 \mu \mathrm{m}$ and a thickness of $150 \mathrm{~nm}$ (Fig. 1b). They have a fibrous morphology, as expected, 

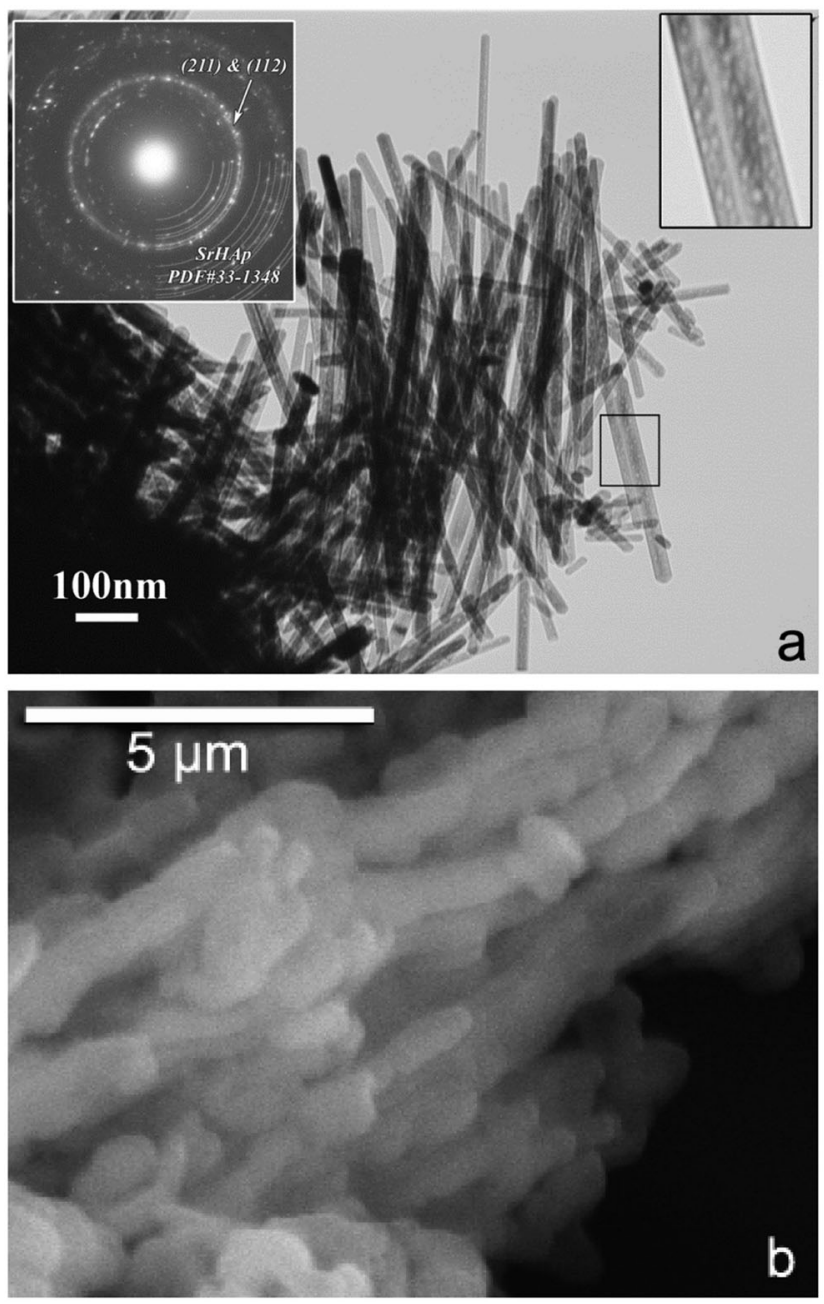

Fig. 1 (a) Conventional TEM bright field (BF) image showing a bundle of SrHNRs. The upper-left inset presents a typical SAED pattern of the material, whereas the upper-right one shows a magnified part of the nanorods revealing the presence of mesopores on the material. (b) SEM micrographs of silica nanotubes.

and tend to aggregate into bigger structures, which is logical because of the presence of hydroxyl groups easily creating hydrogen bonds.

Both of these nanoparticles have been added in the PBSu matrix in order to study their effect on enzymatic hydrolysis and cell growth for tissue engineering applications. ${ }^{23}$ In the present work we have focused on their effect on thermal decomposition kinetics and mechanism of PBSu.

\subsection{Thermal degradation of PBSu, PBSu-SiNTs and PBSU-SrHNRs}

Thermal degradation of PBSu and the effect of SiNTs and SrHNRs on the thermal degradation of PBSu nanocomposites were studied by determining their mass loss upon heating in a TG apparatus. In Fig. 2 the mass (\%) and the derivative of mass loss (DTG) are shown for the two sets of samples. The experiments were conducted at a heating rate of $20{ }^{\circ} \mathrm{C} \mathrm{min}{ }^{-1}$ under a nitrogen atmosphere. As it can be seen in Fig. 2a, the addition

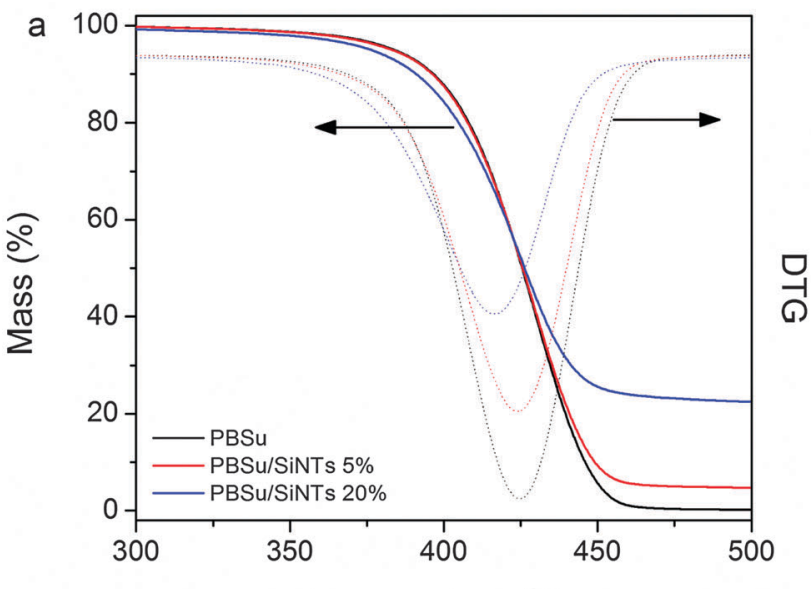

Temperature $\left({ }^{\circ} \mathrm{C}\right)$

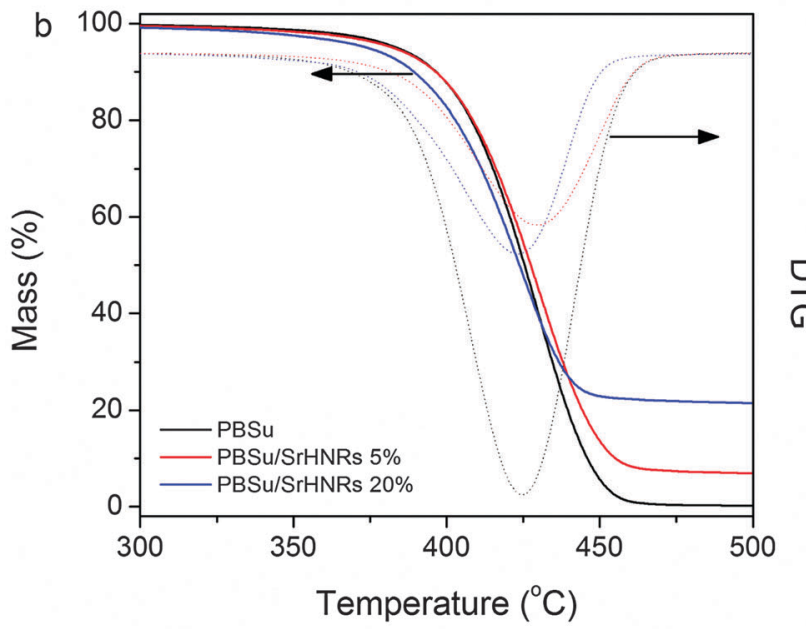

Fig. 2 Mass (\%) and DTG versus temperature obtained from the thermogravimetric experiments for PBSu and its nanocomposites with (a) SiNTs and (b) SrHNRs.

of $5 \mathrm{wt} \%$ SiNTs does not affect the thermal stability of the polymeric matrix and the nanotubes act as inert fillers, while the presence of $20 \mathrm{wt} \%$ SiNTs reduces the thermal stability of the final product. The temperatures which correspond to $2 \%$ mass loss of the studied samples are the same for PBSu and $5 \mathrm{wt} \% \mathrm{PBSu}-\mathrm{SiNTs}$ (about $363^{\circ} \mathrm{C}$ ) while for the nanocomposites with $20 \mathrm{wt} \%$ SiNTs the specific temperature is reduced to $345{ }^{\circ} \mathrm{C}$. Also, the remaining residues of the samples indicate the successful incorporation of the silica nanotubes at the original concentrations. From the DTG curves it can be observed that PBSu and $5 \mathrm{wt} \% \mathrm{PBSu}-\mathrm{SiNT}$ have their highest decomposition rate at the same temperature $\left(424{ }^{\circ} \mathrm{C}\right)$, while the $20 \mathrm{wt} \% \mathrm{PBSu}-\mathrm{SiNTs}$ nanocomposite decomposes more quickly at lower temperature $\left(416^{\circ} \mathrm{C}\right)$. This is another indication that the high percentage of the filler catalyzes the decomposition of the nanocomposites and this phenomenon can be attributed to the presence of a lot of surface silanol groups in SiNTs, as was found by FTIR spectroscopy. ${ }^{23}$

The thermal degradation of $\mathrm{PBSu}-\mathrm{SrHNR}$ nanocomposites can be seen in Fig. 2b. Once again, it seems that the nanorods did not induce an improvement in the thermal stability of the nanocomposites for both concentrations. This time the mass 
loss (mass loss $\approx 2 \%$ ) begins at $363{ }^{\circ} \mathrm{C}$ for neat $\mathrm{PBSu}$ and at lower temperatures for both nanocomposites; for $5 \mathrm{wt} \%$ SrHNRs it is $356{ }^{\circ} \mathrm{C}$, while for $20 \mathrm{wt} \%$ SrHNRs it is $341.5{ }^{\circ} \mathrm{C}$, nearly $21{ }^{\circ} \mathrm{C}$ lower than the neat polymer. In addition, as in the case of SiNTs, strontium nanorods also have a lot of surface hydroxyl groups that could accelerate the decomposition of PBSu and affect negatively the thermal stability of its nanocomposites. Such a behavior regarding nanoparticles containing hydroxyl groups was also reported for other aliphatic polyesters in the literature. ${ }^{24-26}$ However, the maximum decomposition rate of $\mathrm{PBSu}$ and $20 \mathrm{wt} \% \mathrm{PBSu}-\mathrm{SrHNRs}$ is the same since the peak of DTG is at the same temperature of $424{ }^{\circ} \mathrm{C}$, while for $5 \mathrm{wt} \% \mathrm{PBSu}-\mathrm{SrHNRs}$ the DTG peak is at $429{ }^{\circ} \mathrm{C}$. So, it seems that SrHNRs are mainly catalyzing the initial decomposition steps of PBSu.

\subsection{Kinetic analysis}

In order to understand further the results obtained from thermogravimetric experiments and also to observe further aspects of the thermal decomposition of the studied nanocomposites, a kinetic analysis of the thermal degradation process was performed. In order to calculate the values of the kinetic triplet, which includes the activation energy $E$, the preexponential factor $A$ and the reaction model $f(\alpha)$, isoconversional and model-fitting methods for the kinetic analysis were used. During this study, the equations described previously in the "Kinetic methods for thermogravimetric analysis" section were used. Initially, isoconversional methods were applied, which are considered "model free" and assume that the conversion function $f(\alpha)$ does not change with the variation of the heating rate for the different values of the degree of conversion $(\alpha) \cdot{ }^{27}$ Isoconversional methods are considered to give a reliable estimation of the activation energy of thermally stimulated complex processes ${ }^{28}$ and for this reason the differential method proposed by Friedman and the integral method proposed by Kissinger, Akahira and Sunose were applied. The results of the two isoconversional methods are presented in Fig. 3.

As is obvious from Fig. 3, the nanocomposite samples containing $5 \mathrm{wt} \%$ filler content present activation energy values relatively close to the ones calculated for neat PBSu for both isoconversional methods. Furthermore, the samples filled with $20 \mathrm{wt} \%$ filler content present higher differences than neat PBSu, exhibiting significantly lower activation energy values. This can be explained if we take into account that the thermal stability of the samples with highest amount of fillers is significantly lower than that of the neat polymer, as was found by TGA previously. The activation energy findings concerning neat PBSu are in good agreement with previously published results based on the isoconversional method of Friedman. ${ }^{29,30}$ Furthermore, from this study it is clear that both nanofillers can accelerate the decomposition of PBSu but in a different way. Nanocomposites containing SrHNRs have lower activation energies than those corresponding to SiNTs for both used filler contents ( 5 and $20 \mathrm{wt} \%$ ) and thus it can be concluded that they have a stronger effect on the thermal decomposition of PBSu. This could be attributed to the higher amount of available
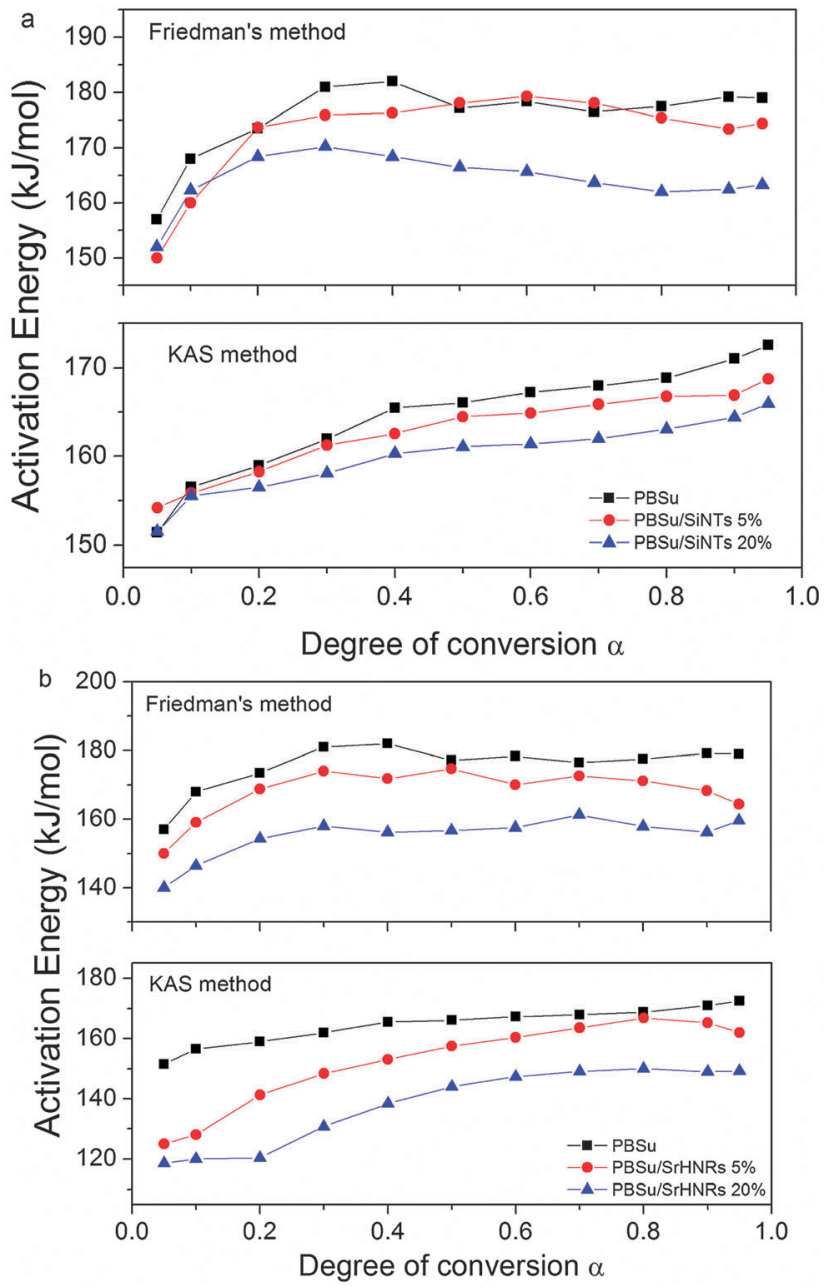

Fig. 3 Dependence of the activation energy $(E)$ on the degree of conversion $(\alpha)$, as calculated using Friedman and KAS methods for (a) PBSu and PBSuSiNT nanocomposites and (b) PBSu and PBSu-SrHNR nanocomposites.

surface hydroxyl groups in SrHNRs, since from contact angle measurements of both nanocomposites it was found that PBSu-SrHNRs have lower contact angles compared to PBSuSiNTs nanocomposites. ${ }^{23}$ Also, from Fig. 3 it can be deduced that the dependence of the activation energy on the degree of conversion can be divided into two different regions for both sets of samples. The first region is extended up to $\alpha=0.3-0.4$ where the activation energy increases rapidly and corresponds to a small mass loss and after that point it remains stable for the rest of the reaction and corresponds to the main degradation mechanism. This dependence of $E$ on $\alpha$ indicates that at least two mechanisms should be employed in order to describe the thermal degradation of PBSu and nanocomposites. The mean activation energy values calculated using Friedman's method are higher than those calculated using the KAS method for all samples. The variation of the activation energy with the degree of conversion is an indication of a complicated reaction and the thermal degradation of polymers is considered complex since they involve a variety of reactions during heating. 


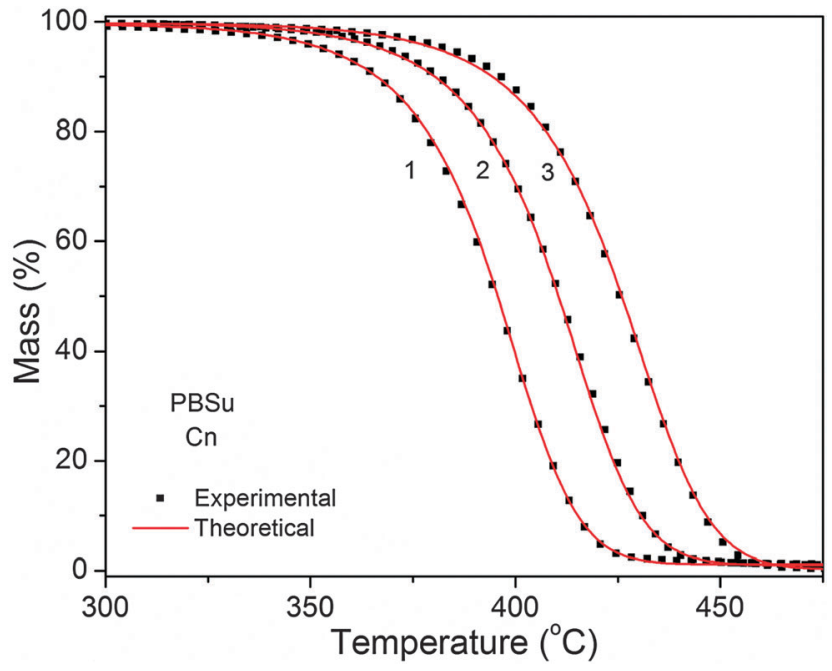

Fig. 4 Thermal degradation of PBSu at different heating rates $\left(1: 5^{\circ} \mathrm{C}\right.$ $\mathrm{min}^{-1}, 2: 10{ }^{\circ} \mathrm{C} \mathrm{min}^{-1}$, and $3: 20{ }^{\circ} \mathrm{C} \mathrm{min}^{-1}$ ). The black signs represent the experimental values and the red line represents the single-step fitting with an $n$-th order model with autocatalysis (Cn).

In order to identify this complexity and the existed mechanisms, the second step of the kinetic analysis involves the modelfitting method. ${ }^{31,32}$ During this process the experimental data are compared with the simulated ones, which occur from the use of different kinetic models. During the model-fitting process, the samples were heated with 3 different heating rates $\left(5,10,20{ }^{\circ} \mathrm{C} \min ^{-1}\right)$. The dependence of thermal degradation upon the heating rate is demonstrated in Fig. 4 where the neat PBSu sample is fitted with a single step mechanism, for which we assume that it corresponds to the main mass loss of the polymer during heating. The reaction model which gave the best fitting results was the $n$-th order model with autocatalysis $(\mathrm{Cn})$ for which the conversion function equals: $f(\alpha)=(1-\alpha)^{n}\left(1+k_{\text {cat }} \alpha\right)$.

As it can be seen from Fig. 4 the correlation between the experimental and theoretical values is very good and the correlation coefficient is high $\left(R^{2}=0.999\right)$ for the neat PBSu sample. The values of the activation energy and the preexponential factor along with the calculated values from the single-step fitting with an $n$-th order model with autocatalysis of the nanocomposites are presented in Table 1 . The calculated values for the activation energy are close to the ones obtained from the isoconversional methods, so this is another indication of the accuracy of the results. ${ }^{33,34}$ Also, from this study it was proved once again that both nanofillers accelerate PBSu

Table 1 Activation energy, pre-exponential factor, reaction order and correlation coefficient after fitting with a single step mechanism and using the $n$-th order with autocatalysis $(\mathrm{Cn})$ reaction model

\begin{tabular}{llrll}
\hline Material & $\begin{array}{l}\text { Activation energy } \\
\left(\mathrm{kJ} \mathrm{mol}^{-1}\right)\end{array}$ & $\begin{array}{l}\log \mathrm{A} \\
\left(\mathrm{s}^{-1}\right)\end{array}$ & $\begin{array}{l}\text { Reaction } \\
\text { order }\end{array}$ & $\begin{array}{l}\text { Correlation } \\
\text { coefficient }\end{array}$ \\
\hline PBSu & 172.7 & 10.7 & 1.1 & 0.9997 \\
PBSu-SiNTs 5 wt\% & 166.1 & 10.2 & 1.1 & 0.9997 \\
PBSu-SiNTs 20 wt\% & 157.4 & 10.2 & 1.3 & 0.9998 \\
PBSu-SrHNRs 5 wt\% & 164.7 & 9.1 & 1 & 0.9997 \\
PBSu-SrHNRs 20 wt\% & 152.5 & 10.4 & 1.2 & 0.9997
\end{tabular}

thermal degradation but SrHNRs affect this process more drastically than SiNTs since for both concentrations the corresponding activation energies are slightly lower. Other models
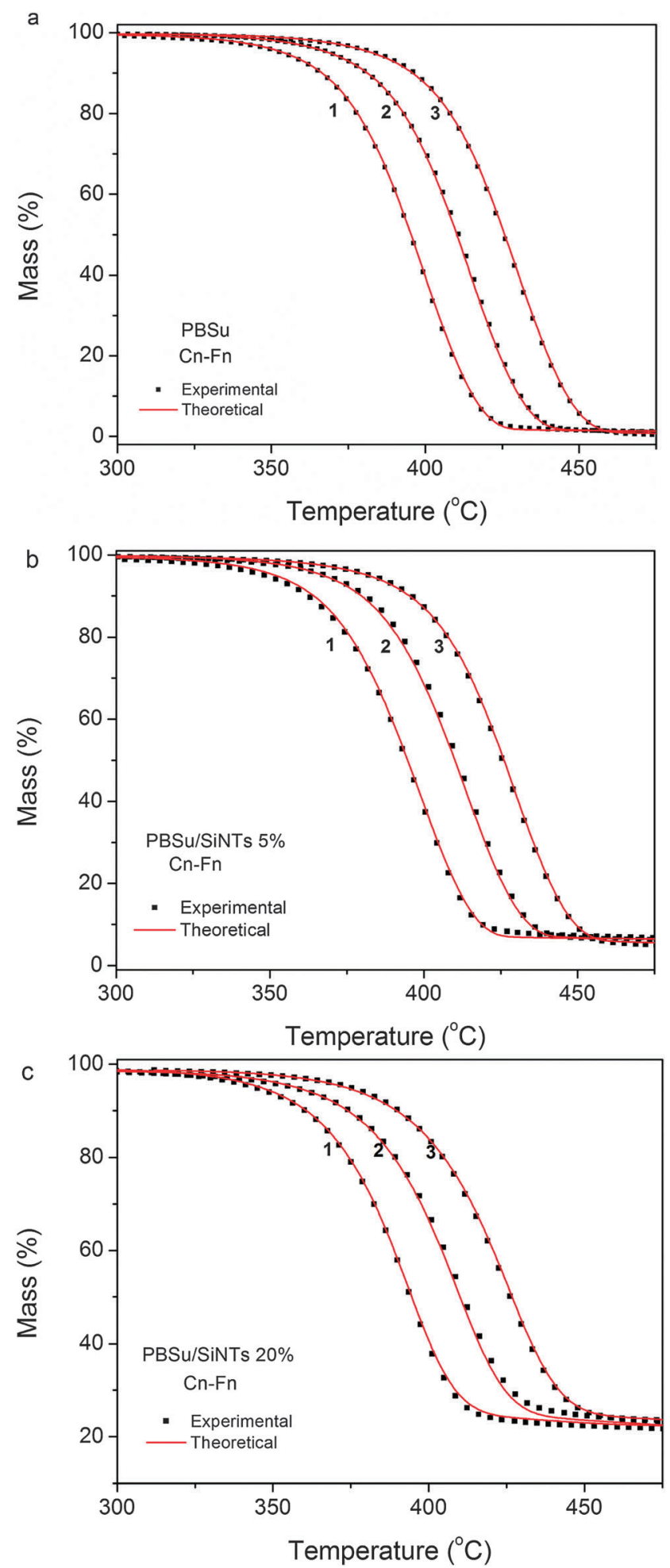

Fig. 5 Thermal degradation of PBSu (a), nanocomposites with 5 wt\% PBSuSiNTs (b), and $20 \mathrm{wt} \%$ PBSu-SiNTs (c) at different heating rates $\left(1: 5^{\circ} \mathrm{C} \mathrm{min}^{-1}\right.$, 2: $10{ }^{\circ} \mathrm{C} \mathrm{min}^{-1}$, and $3: 20{ }^{\circ} \mathrm{C} \mathrm{min}^{-1}$ ). The black signs represent the experimental values and the red line represents the dual consecutive step fitting. 

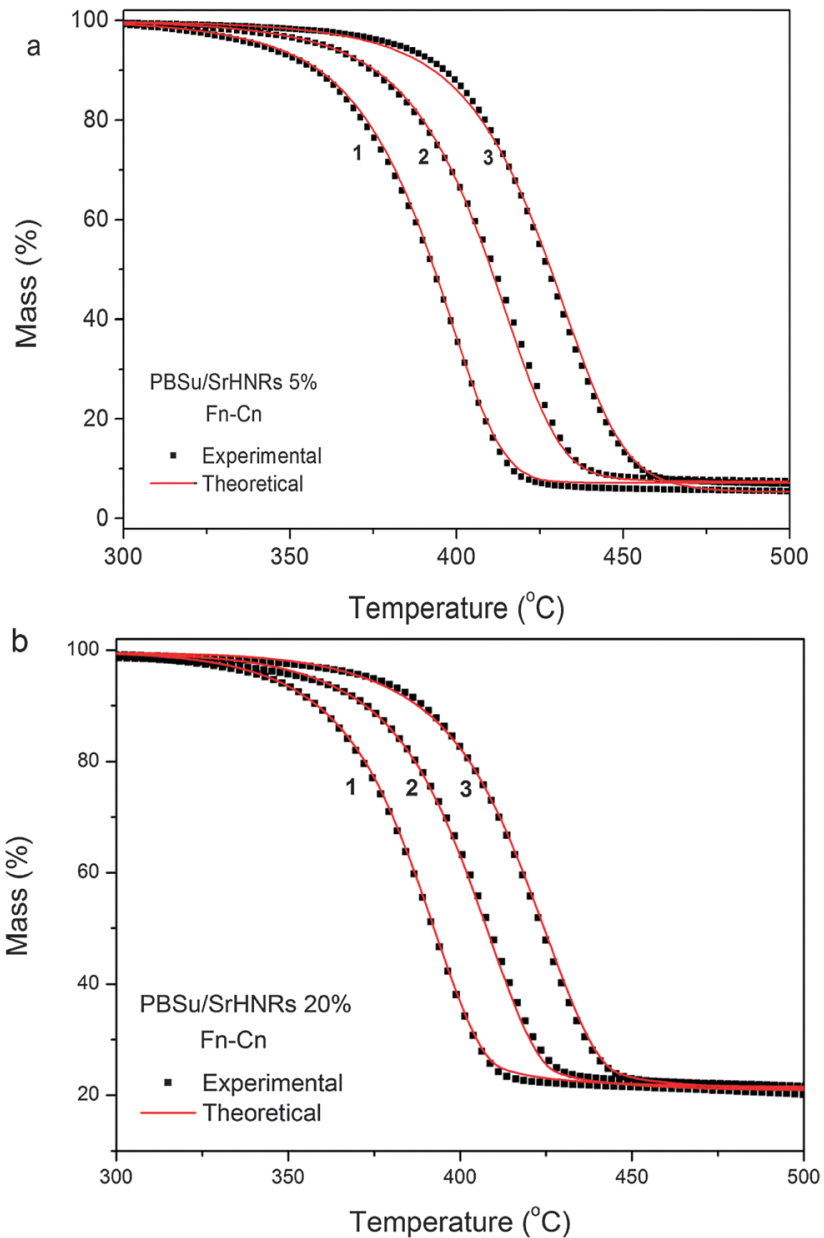

Fig. 6 Thermal degradation of (a) 5 wt\% PBSu-SrHNRs and (b) 20 wt\% PBSu-SrHNRs at different heating rates $\left(1: 5^{\circ} \mathrm{C} \mathrm{min}^{-1}, 2: 10^{\circ} \mathrm{C} \mathrm{min}^{-1}\right.$, and 3: $\left.20^{\circ} \mathrm{C} \mathrm{min}^{-1}\right)$. The black signs represent the experimental values and the red line represents the dual consecutive step fitting.

were also tested on their applicability but their correlation coefficient values were quite low and the estimated values were considered inaccurate.

As it was stated earlier, the thermal degradation of polymer nanocomposites is a rather complex phenomenon, so the presence of two consecutive or parallel models during the fitting of experimental and theoretical values should be thoroughly examined for the calculation of activation energy and pre-exponential factor. The assumptions that were made prior to the determination of the thermal degradation mechanism were mainly two: the two mechanisms were consecutive and the first mechanism corresponds to a small mass loss according to the experimental results. Different combinations of the 16 applied models were tested and the one that gave the most accurate fitting results and values for the samples containing 5 and $20 \mathrm{wt} \%$ silica nanotubes and $20 \mathrm{wt} \%$ SrHNRs was the two consecutive step reaction with $n$-th order with autocatalysis (Cn) $\left[f(\alpha)=(1-\alpha)^{n}\left(1+k_{\text {cat }} \alpha\right)\right]$ and $n$-th order (Fn) $[f(\alpha)=$ $\left.(1-\alpha)^{n}\right]$ models. In contrast, the one that was applied successfully to the sample containing $5 \mathrm{wt} \%$ SrHNRs was the $n$-th order (Fn) and $n$-th order model with autocatalysis (Cn). The results from the fitting are presented in Fig. 5 and 6 .

As is obvious, the correlation between the experimental and theoretical values for all samples under study was very good except for very small deviations observed at the end of the degradation process in the nanocomposites. The calculated values of the activation energy are similar to the values obtained by the isoconversional methods of Friedman and KAS and they are presented in Table 2. The variation of the kinetic parameters reflects the change in the degradation mechanism and serves as another indication that the use of two mechanisms is necessary for the simulation of the degradation process with mathematical models. The conclusions that were stated earlier regarding the activation energy values are once again confirmed, since PBSu presents higher activation energies in both mechanisms than the nanocomposite samples. Furthermore, the fact that SrHNRs are mainly catalyzing the initial decomposition steps of PBSu is obvious here, since the activation energies of the first mechanism, which corresponds to the start of the decomposition process, are significantly lower than PBSu. The high percentage of surface hydroxyl groups which are included into SrHNRs decompose at low temperatures, thereby attributing low activation energies to the nanocomposite samples. Moreover, the change in the mechanisms which describe the decomposition of PBSu-SrHNRs is an

Table 2 Activation energy, pre-exponential factor, reaction order and correlation coefficient after fitting with a two consecutive step mechanism using the $n$-th order with autocatalysis $(\mathrm{Cn})$ and $n$-th order $(\mathrm{Fn})$ reaction models

\begin{tabular}{|c|c|c|c|c|c|}
\hline PBSu & 1st (Cn) & 166.9 & 10.2 & 0.7 & 0.9998 \\
\hline PBSu-SiNTs 20 wt $\%$ & & 150.2 & 10 & 0.9 & 0.9997 \\
\hline PBSu & 2nd (Fn) & 175.5 & 9.7 & 1 & 0.9998 \\
\hline PBSu-SiNTs 5 wt\% & & 168.4 & 9.5 & 1.9 & 0.9998 \\
\hline PBSu-SrHNRs 5 wt\% & 1st (Fn) & 138.1 & 8.1 & 1.1 & 0.9998 \\
\hline PBSu-SrHNRs 20 wt $\%$ & & 132.2 & 10.1 & 0.7 & 0.9997 \\
\hline PBSu-SrHNRs 5 wt $\%$ & 2nd (Cn) & 165.4 & 11.3 & 0.9 & 0.9998 \\
\hline PBSu-SrHNRs $20 \mathrm{wt} \%$ & & 157.7 & 12.9 & 1.7 & 0.9997 \\
\hline
\end{tabular}



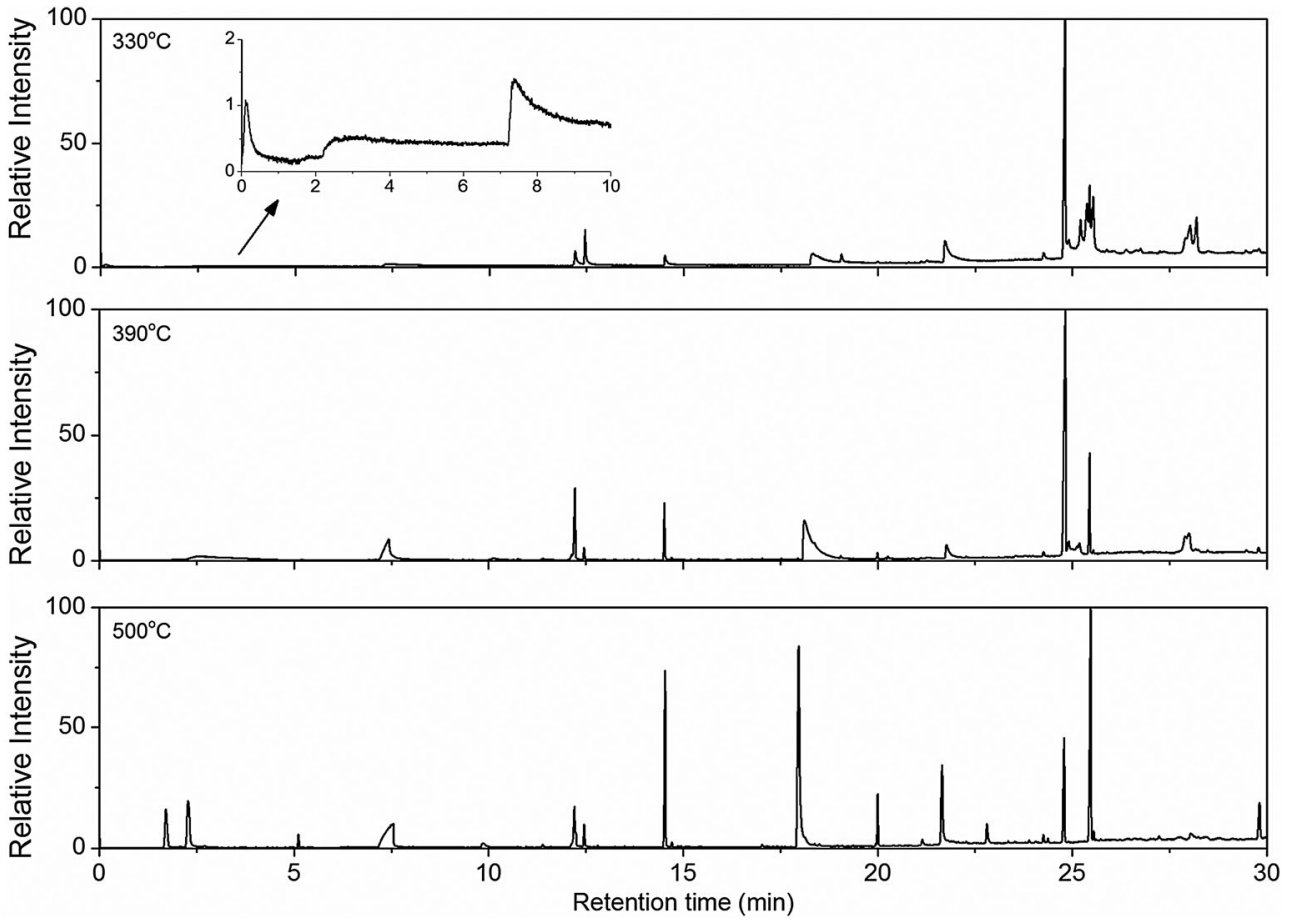

Fig. 7 Chromatograms of PBSu at 330,390 and $500{ }^{\circ} \mathrm{C}$.
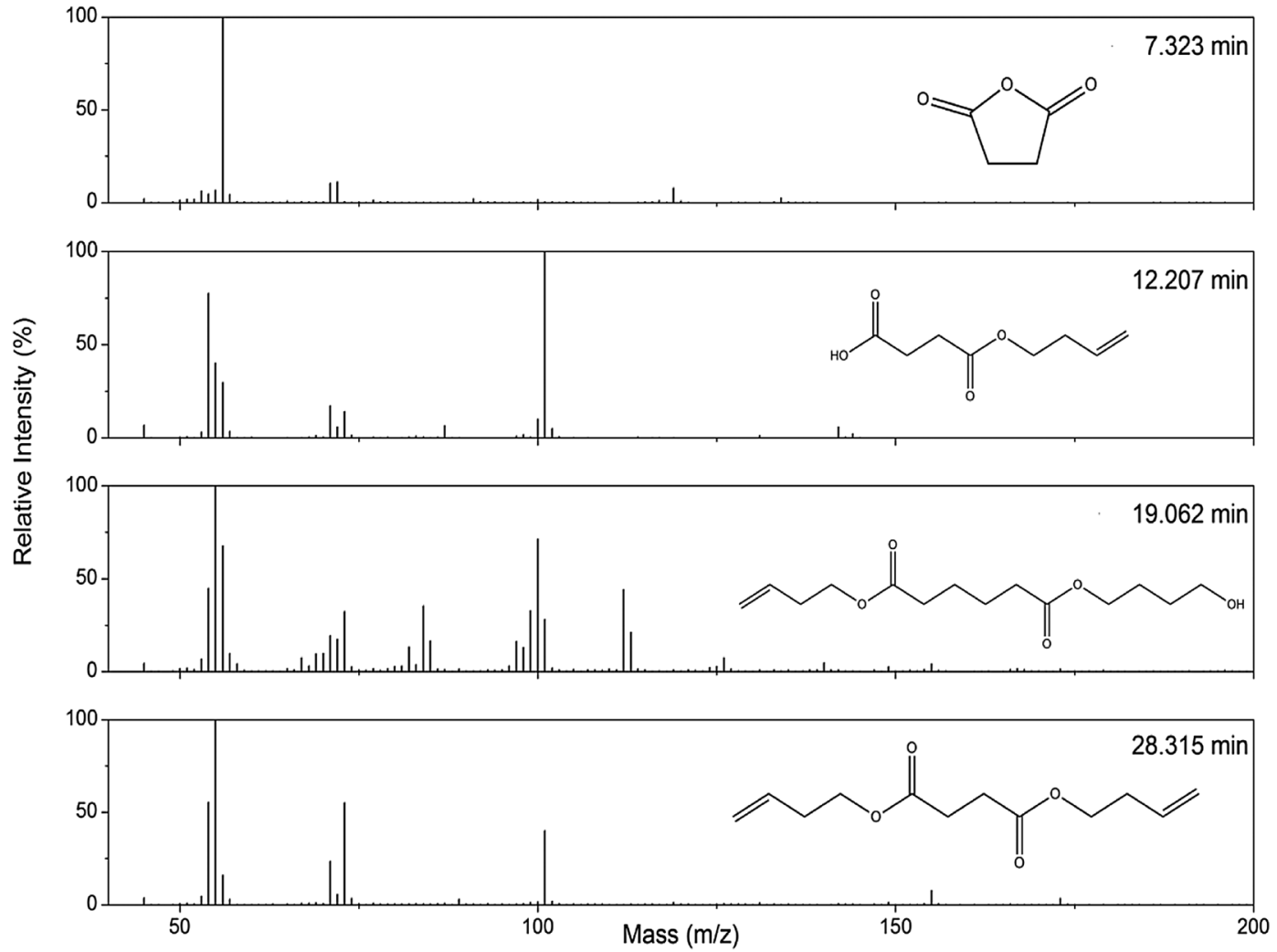

Fig. 8 Mass to charge ratios for the chromatogram peaks at 7.323, 12.201, 19.207 and 28.315 min of neat PBSu collected during pyrolysis at $330{ }^{\circ} \mathrm{C}$.

indication that the specific filler modifies to an extent the degradation of the nanocomposite sample, thus the detailed examination of the decomposition by Py-GC-MS would be very useful.
Regarding the samples filled with SiNTs, the small differences in the activation energy values and the fact that the mechanism that describes the degradation process is the same are an 
Table 3 Decomposition products of PBSu

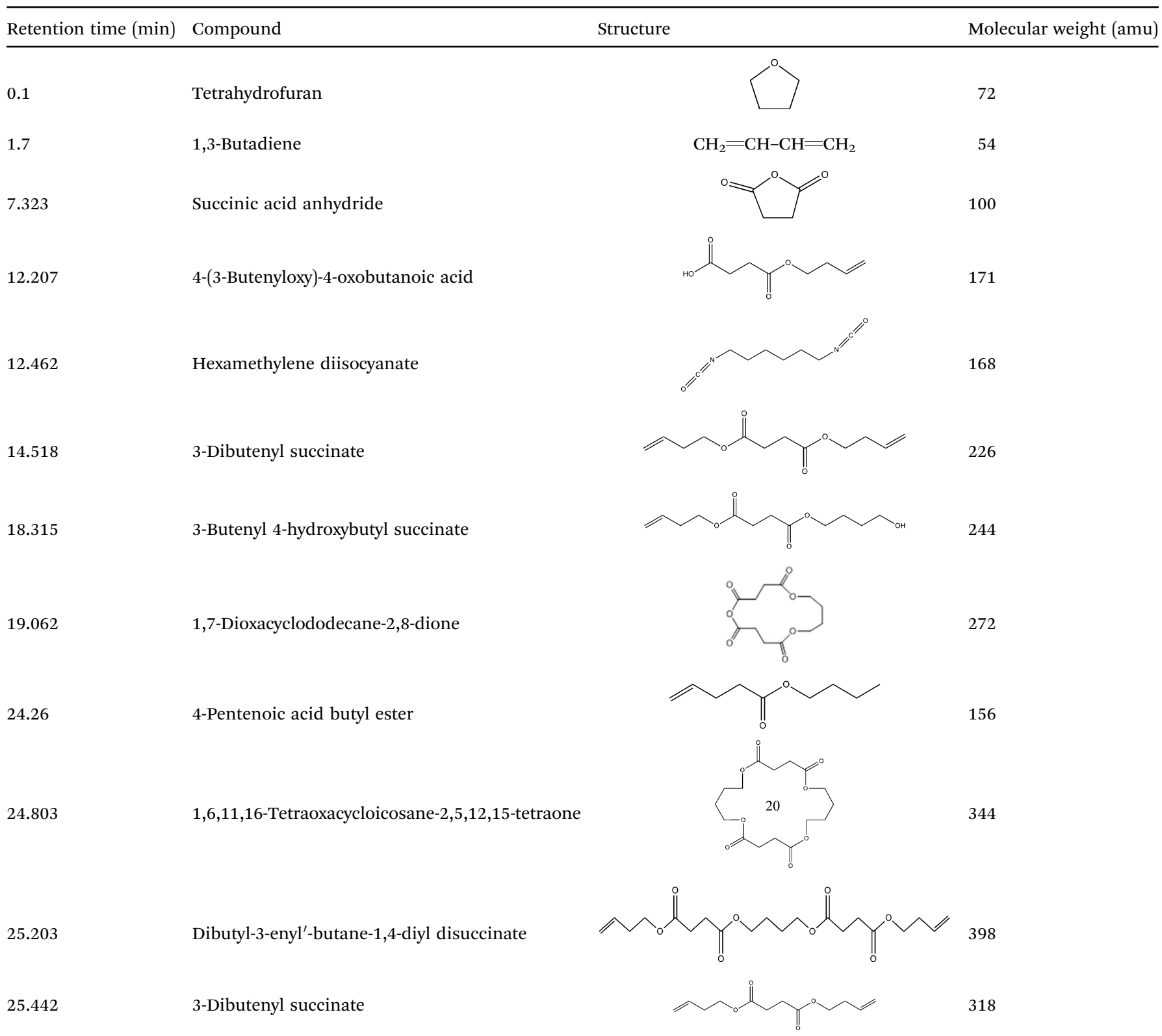

At higher retention times, higher allyl and diallyl succinates were collected.

indication that SiNTs affect to a significantly smaller extent the decomposition of the polymeric matrix than SrHNRs, even at high filler content.

\subsection{Evaluation of evolved gas analysis by GC-MS}

From the above measurements it is clear that both nanofillers can affect negatively the thermal stability of PBSu but in a different way. Apart from the catalyzing effect of the used nanofillers, in some cases it was reported that nanofillers can also affect the decomposition mechanism of polyesters and formed products. ${ }^{35,36}$ In order to evaluate this in our samples Py-GC-MS was used. In Fig. 7 the chromatograms of neat PBSu collected at 330,390 and $500{ }^{\circ} \mathrm{C}$ are presented, which corresponds to the initial, middle and final decomposition step temperatures. As it can be seen, the evolution patterns from the 10-12th min until the end of retention time are essentially the same with differences only in the intensity of the products. As the decomposition temperature increased, the intensity of the formed byproducts after a retention time of 12th $\mathrm{min}$ increased too and only at $500{ }^{\circ} \mathrm{C}$ the intensity for a retention time of $24.8 \mathrm{~min}$ was reduced.

From the collected mass spectra of each chromatograph peak (some characteristic spectra are presented in Fig. 8), it was revealed that the first gaseous products evolving through $\mathrm{PBSu}$ decomposition were tetrahydrofuran, 1,3-butadiene and succinic acid anhydride, detected along with water and $\mathrm{CO}$ or $\mathrm{CO}_{2}$ during the first 3.5 and 7 min of retention respectively. Similar decomposition products, except succinic anhydride, were also 


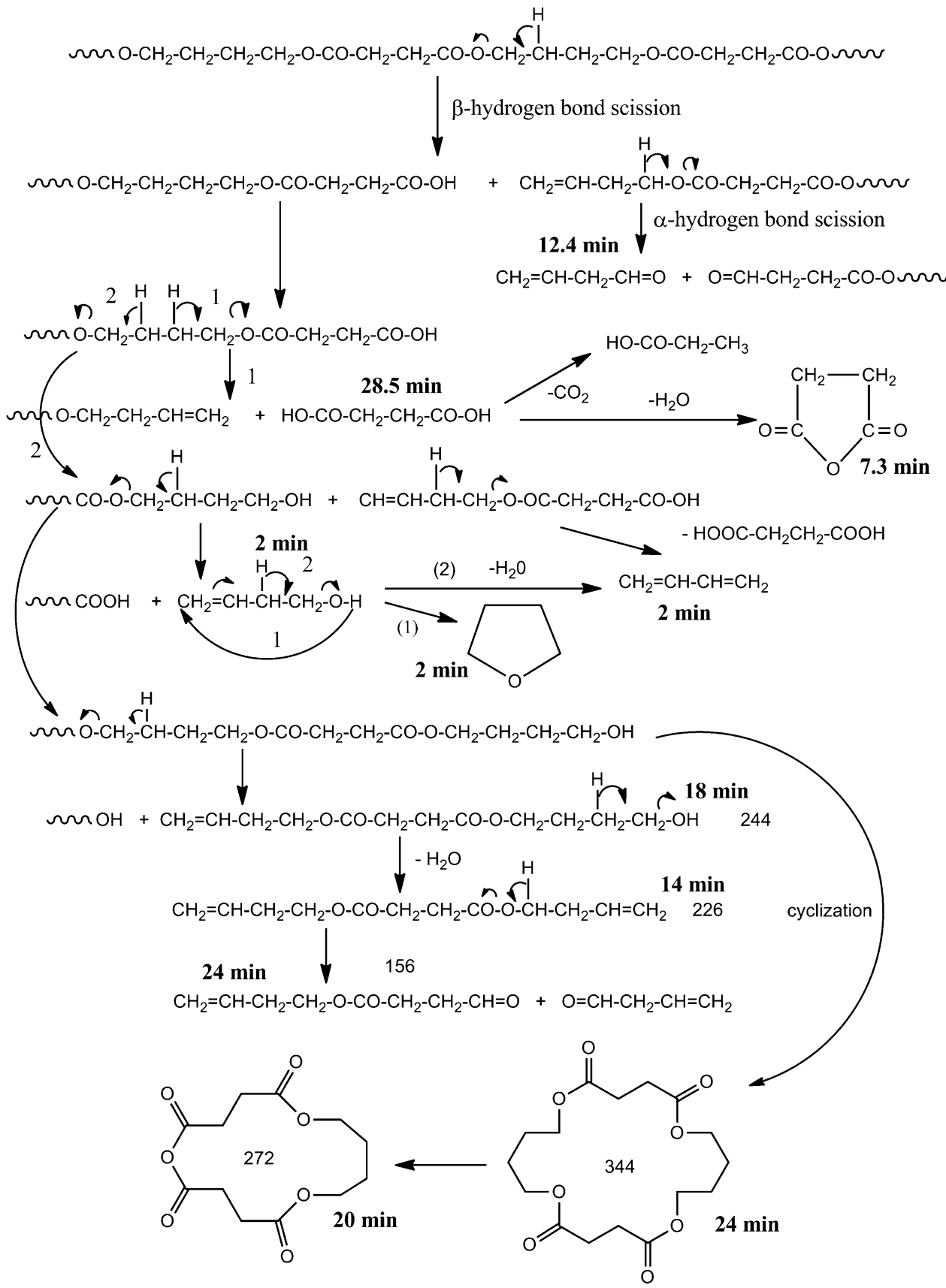

Fig. 9 Decomposition mechanism of PBSu and formed byproducts.

observed during decomposition of poly(butylene terephthalate) (PBT) and are due to the existence of 1,4-butylene glycol ${ }^{37}$ while $\mathrm{CO}$ and $\mathrm{CO}_{2}$ are due to the carboxyl end groups of polyesters. $^{38,39}$ At higher retention times allyl and diallyl compounds with characteristic vinyl groups and higher molecular weights were detected. Furthermore, it seems that the amount of decomposition products is directly dependent on the decomposition temperature.

In the case of the experiments performed at 330 and $390{ }^{\circ} \mathrm{C}$, the decomposition products were detected in lower amounts, since their peaks are low in intensity, and at $500{ }^{\circ} \mathrm{C}$ the decomposition products were detected in higher amounts due to the increased intensity of the peaks. This is an indication that at higher temperatures decomposition proceeds with much faster rates and byproducts with progressively higher molecular weights are produced. This was also confirmed from our previous studies. ${ }^{40,41}$ In the case of aliphatic polyesters prepared from ethylene or propylene glycol it was found that the first mechanism that takes place at low temperatures is autocatalysis and corresponds to a small mass loss. Volatile products such as $\mathrm{CO}_{2}$, ethylene and propylene are the main products. In the $500{ }^{\circ} \mathrm{C}$ experiment, the first recorded peak around 1.7 min was 1,3-butadiene, the second at $2.3 \mathrm{~min}$ was tetrahydrofuran and the third at 5.1 min was 3-butenylpropionate. From that point 
on, the collected products were exactly the same, they were recorded in the same order and at similar retention times with the only difference being their total intensity though at different pyrolysis temperatures. In Table 3 the decomposition products of neat PBSu are presented along with their molecular weights and the retention times in which they were collected (absolute retention time corresponds to pyrolysis at $330{ }^{\circ} \mathrm{C}$ ).

For higher retention times, as it can be seen from Table 3 the main decomposition products of PBSu are succininic acid, allyl and diallyl compounds, which is in agreement with our previous studies in aliphatic polyesters. ${ }^{40,42}$ Based on the detected compounds, the decomposition mechanism illustrated in Fig. 9 is proposed with the formed products and the corresponding retention times. The decomposition of PBSu and its nanocomposites takes place mainly through $\beta$-hydrogen bond scission which is a six-membered cyclic transition state ( $\beta$-decomposition). ${ }^{42,43}$ From this procedure allyl and diallyl compounds are produced. However, as it can be seen there are also some aldehydes as byproducts, which can be formed through $\alpha$-hydrogen bond scission (retention time $14.2 \mathrm{~min}$ ). Also some cyclic decomposition products can be prepared from the nucleation reaction of hydroxyl end groups with ester backbone groups of macromolecular chains. This cyclization mechanism was also detected in aromatic polyesters like poly(ethylene terephthalate) (PET) and $\mathrm{PBT}^{44}{ }^{4}$ The molecular weight of the recorded cyclic products in PBSu with retention times of 20 and $24 \mathrm{~min}$ is not higher than $334 \mathrm{amu}$ which corresponds to 2 repeating units of succinic acid and butylene glycol.

In Fig. 10a the chromatograms of PBSu and PBSu with 5 wt $\%$ SiNTs, corresponding to 330,390 and $500{ }^{\circ} \mathrm{C}$ experiments, reveal almost no differences caused by the presence of the filler. The recorded products are exactly the same and evolve in the same pattern. Only minor localized differences in the intensity of some decomposition compounds could be distinguished, supporting the decomposition kinetic findings that the presence of $5 \mathrm{wt} \%$ SiNTs does not affect the process. The same also appears for nanocomposites containing $20 \mathrm{wt} \%$ SiNTs (Fig. 10b). The evolving compounds were identical and appeared in the same order as in neat PBSu, indicating that SiNTs do not affect the decomposition mechanism of PBSu.

However, it seems that the addition of SrHNRs as fillers into PBSu causes some effect on its decomposition mechanism. In the chromatograms corresponding to pyrolysis experiments of PBSu and its nanocomposite with $5 \%$ SrHNRs at $330{ }^{\circ} \mathrm{C}$, changes in the evolution pattern due to the presence of the filler, between 24 and $30 \mathrm{~min}$ can clearly be seen (Fig. 11a, inset graph). Some peaks between 25 and $26 \mathrm{~min}$ have disappeared in nanocomposites. Similar differences can be seen in the chromatograms corresponding to 390 and $500{ }^{\circ} \mathrm{C}$ decomposition temperatures as well. However, for these 2 temperatures distinct differences between the neat polymer and its nanocomposite are visible even from the early decomposition peaks recorded in the first 7-9 min of retention and between 12-14 min. Moreover, during the last minutes of retention, between $24-30 \mathrm{~min}$, modifications on the evolution
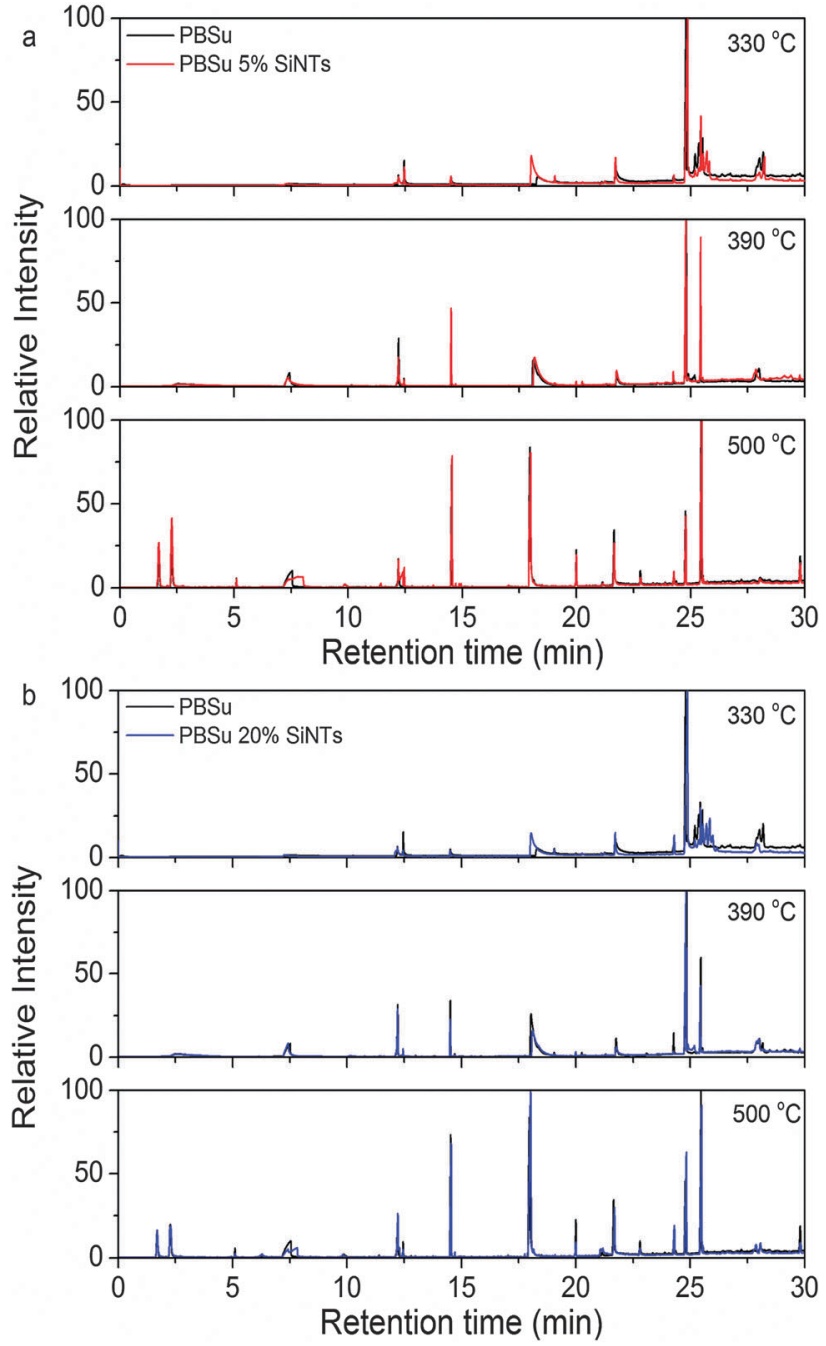

Fig. 10 (a) Chromatograms of PBSu and 5 wt\% PBSu-SiNTs at 330, 390 and $500{ }^{\circ} \mathrm{C}$. (b) Chromatograms of PBSu and 20 wt\% PBSu-SiNTs at 330 , 390 and $500{ }^{\circ} \mathrm{C}$.

profiles are more visible (inset graph). Nevertheless, even though differences in the evolution patterns were detected due to the presence of $5 \mathrm{wt} \%$ SrHNRs, the decomposition products were identical and evolved in the same order as in neat PBSu. This implies that only a minor modification on the decomposition reactions can be justified and since some peaks were not recorded in nanocomposites it can be said that due to the catalyzing effect of SrHNRs the decomposition path of PBSu has been simplified. This suggestion is in agreement with the kinetic findings which revealed that the incorporation of SrHNRs in PBSu induced minor modifications in the decomposition mechanisms (the $n$-th order with autocatalysis was switched to the $n$-th order and vice versa). Similar differences were also recorded when SrHNRs were added in higher amounts (20 wt\%) (Fig. 11b). Some differences in the evolution patterns, mainly after 17 minutes of retention, are obvious in the chromatogram of PBSu containing $20 \%$ SrHNRs. In that case also, no differences in the collected decomposition products or their evolution order 

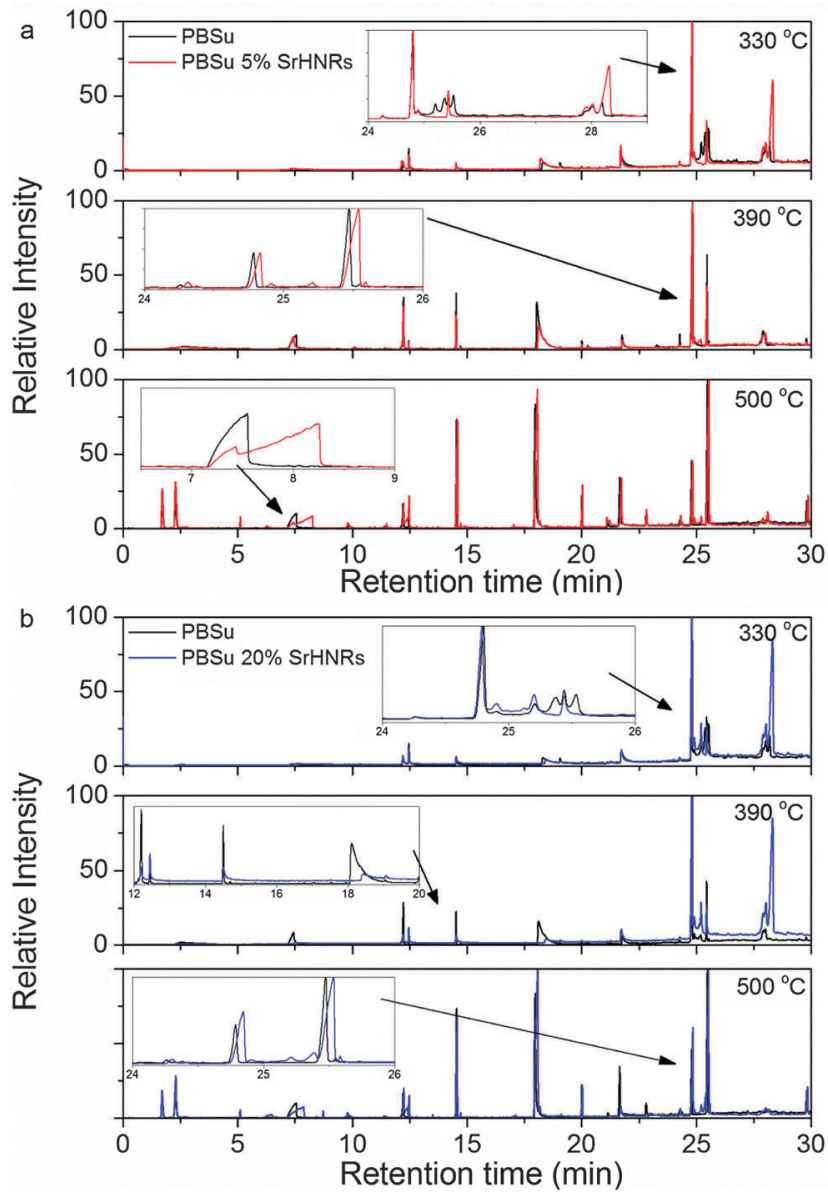

Fig. 11 (a) Chromatograms of PBSu and 5 wt\% PBSu-SrHNRs at 330, 390 and $500{ }^{\circ} \mathrm{C}$, (b) Chromatograms of PBSu and 20 wt\% PBSu-SrHNRs at 330 , 390 and $500{ }^{\circ} \mathrm{C}$.

were detected through the evaluation of collected mass spectra. Therefore, a minor modification corresponding mainly to the final decomposition steps could be justified. This finding supports the kinetic results that proposed that the presence of $20 \mathrm{wt} \%$ SrHNRs modifies the kinetic parameters of the second mechanism.

\section{Conclusions}

PBSu nanocomposites containing 5 and $20 \mathrm{wt} \%$ of SrHNRs and SiNTs were prepared by melt-mixing and the effects of the nanofillers on the thermal stability and decomposition kinetics of PBSu were examined by a combination of thermogravimetry and pyrolysis-gas chromatography-mass spectroscopy. From TGA curves and activation energies it was found that both nanofillers affect the decomposition of PBSu, with SrHNRs exhibiting a higher catalyzing effect, maybe due to their high amount of surface hydroxyl groups. The calculated activation energies are much lower in the nanocomposite samples and they are also dependent on the content of the used filler. From Py-GC-MS and the identified decomposition products it was found that the decomposition of PBSu is taking place mainly via $\beta$-hydrogen scission and to a lower extent with $\alpha$-hydrogen scission. In the first case $\mathrm{CO}, \mathrm{CO}_{2}, \mathrm{H}_{2} \mathrm{O}, 1,3$-butadiene, tetrahydrofuran, succinic acid and its anhydride, and allyl- and diallyl compounds are produced while in the second aldehydes. The decomposition mechanism is also temperature-dependent since larger amounts of high molecular weight compounds are produced at higher degradation temperatures. In PBSu-SiNT nanocomposites the evolving compounds were identical and appeared in the same order as in neat PBSu, indicating that SiNTs do not affect the decomposition mechanism of PBSu. However, it seems that the addition of SrHNRs into PBSu causes a minor effect on its decomposition mechanism since there are some differences in the retention times of evolved products, a fact which was once again confirmed by the findings from thermogravimetric analysis.

\section{Acknowledgements}

The authors wish to acknowledge co-funding of this research by IKY (Greece) and DAAD (Germany), Action "IKYDA 2012".

\section{References}

1 H. Wang, J. Ji, W. Zhang, Y. Zhang, J. Jiang, Z. Wu, S. Pu and P. K. Chu, Acta Biomater., 2009, 5, 279-287.

2 J. Jagur-Grodzinski, Polym. Adv. Technol., 2006, 17, 395-418. 3 H. M. Wong, K. W. K. Yeung, K. O. Lam, V. Tam, P. K. Chu, K. D. K. Luk and K. M. C. Cheung, Biomaterials, 2010, 31, 2084-2096.

4 H. Shirahama, Y. Kawaguchi, M. S. Aludin and H. Yasuda, J. Appl. Polym. Sci., 2001, 80, 340-347.

5 M. Ishii, M. Okazaki, Y. Shibasaki, M. Ueda and T. Teranishi, Biomacromolecules, 2001, 2, 1267-1270.

6 P. Rizzarelli and S. Carroccio, Polym. Degrad. Stab., 2009, 94, 1825-1838.

7 Y. Zhao, J. Qu, Y. Feng, Z. Wu, F. Chen and H. Tang, Polym. Adv. Technol., 2012, 23, 632-638.

8 D. N. Bikiaris, Polym. Degrad. Stab., 2013, 98, 1908-1928.

9 B. Sitharaman, X. Shi, X. F. Walboomers, H. Liao, V. Cuijpers, L. J. Wilson, A. G. Mikos and J. A. Jansen, Bone, 2008, 43, 362-370.

10 X. Shi, J. L. Hudson, P. P. Spicer, J. M. Tour, R. Krishnamoorti and A. G. Mikos, Biomacromolecules, 2006, 7, 2237-2242.

11 G. Lalwani, A. M. Henslee, B. Farshid, L. Lin, F. K. Kasper, Y.-X. Qin, A. G. Mikos and B. Sitharaman, Biomacromolecules, 2013, 14, 900-909.

12 J. Panyam and V. Labhasetwar, Adv. Drug Delivery Rev., 2003, 55, 329-347.

13 C. Zhang, C. Li, S. Huang, Z. Hou, Z. Cheng, P. Yang, C. Peng and J. Lin, Biomaterials, 2010, 31, 3374-3383.

14 K. R. Martin, J. Nutr., Health Aging, 2007, 11, 94-97.

15 M.-H. Kim, Y.-J. Bae, M.-K. Choi and Y.-S. Chung, Biol. Trace Elem. Res., 2009, 128, 239-247.

16 K. Chrissafis and D. Bikiaris, Thermochim. Acta, 2011, 523, $1-24$. 
17 D. Bikiaris, Thermochim. Acta, 2011, 523, 25-45.

18 F. Marlow and F. Kleitz, Microporous Mesoporous Mater., 2001, 44-45, 671-677.

19 F. Kleitz, F. Marlow, G. D. Stucky and F. Schüth, Chem. Mater., 2001, 13, 3587-3595.

20 H. L. Friedman, J. Polym. Sci., Part C: Polym. Symp., 1964, 6, 183-195.

21 H. E. Kissinger, Anal. Chem., 1957, 29, 1702-1706.

22 T. Akahira and T. Sunose, Res. Report Chiba Inst. Technol. (Sci. Technol.), 1971, 16, 22-31.

23 I. Grigoriadou, N. Nianias, A. Hoppe, Z. Terzopoulou, D. Bikiaris, J. Will, J. Hum, J. A. Roether, R. Detsch and A. R. Boccaccini, Composites, Part B, 2014, 60, 49-59.

24 K. Chrissafis, G. Antoniadis, K. M. Paraskevopoulos, A. Vassiliou and D. N. Bikiaris, Compos. Sci. Technol., 2007, 67, 2165-2174.

25 P. Bordes, E. Pollet, S. Bourbigot and L. Avérous, Macromol. Chem. Phys., 2008, 209, 1473-1484.

26 M. Erceg, T. Kovačić and S. Perinović, Thermochim. Acta, 2008, 476, 44-50.

27 S. Vyazovkin, J. Therm. Anal. Calorim., 2006, 83, 45-51.

28 A. Pratap, T. Lilly Shanker Rao, K. N. Lad and H. D. Dhurandhar, J. Therm. Anal. Calorim., 2007, 89, 399-405.

29 K. Chrissafis, K. M. Paraskevopoulos and D. N. Bikiaris, Thermochim. Acta, 2005, 435, 142-150.

30 S.-F. Lu, M. Chen and C. H. Chen, J. Appl. Polym. Sci., 2012, 123, 3610-3619.
31 S. Vyazovkin and C. A. Wight, Thermochim. Acta, 1999, 340-341, 53-68.

32 S. Vyazovkin and N. Sbirrazzuoli, Macromol. Rapid Commun., 2006, 27, 1515-1532.

33 D. G. Papageorgiou, D. N. Bikiaris and K. Chrissafis, Thermochim. Acta, 2012, 543, 288-294.

34 A. K. Burnham, Thermochim. Acta, 2000, 355, 165-170.

35 J. Zhang, Q. Ji, P. Zhang, Y. Xia and Q. Kong, Polym. Degrad. Stab., 2010, 95, 1211-1218.

36 M. C. Costache, D. D. Jiang and C. A. Wilkie, Polymer, 2005, 46, 6947-6958.

37 R. M. Lum, J. Polym. Sci., Polym. Chem. Ed., 1979, 17, 203-213.

38 F. D. Kopinke, M. Remmler, K. Mackenzie, M. Möder and O. Wachsen, Polym. Degrad. Stab., 1996, 53, 329-342.

39 J. L. Atkinson and S. Vyazovkin, Macromol. Chem. Phys., 2012, 213, 924-936.

40 D. N. Bikiaris, K. Chrissafis, K. M. Paraskevopoulos, K. S. Triantafyllidis and E. V. Antonakou, Polym. Degrad. Stab., 2007, 92, 525-536.

41 K. Chrissafis, K. M. Paraskevopoulos and D. Bikiaris, Thermochim. Acta, 2010, 505, 59-68.

42 K. Chrissafis, E. Roumeli, K. M. Paraskevopoulos, N. Nianias and D. N. Bikiaris, J. Anal. Appl. Pyrolysis, 2012, 96, 92-99.

43 M. Chan Sin, S. N. Gan, M. S. Mohd Annuar and I. K. Ping Tan, Polym. Degrad. Stab., 2010, 95, 2334-2342.

44 F. Samperi, C. Puglisi, R. Alicata and G. Montaudo, Polym. Degrad. Stab., 2004, 83, 3-10. 